different from that of mammals, operates. V. A. Tucker, also from Duke, explained the energetics of bird flight-a field in which a knowledge of aerodynamics and physiology combined with excellent experimentation involving trained birds in a wind-tunnel has led to a real understanding. E. Skadhauge (University of Copenhagen) who was not only responsible for demonstrating unequivocally that salts and water from urine can be reabsorbed in the cloaca and rectum, but also for describing the transport mechanisms involved, has profitably extended his work to study the physiological adaptations of this process in relation to habitat.

Much of the work presented was of fundamental importance to an understanding of animal organisation, for example, M. Peaker (ARC Institute of Animal Physiology, Babraham) presented evidence that the osmoreceptors which control the salt glands in marine birds are located in or near the heart, and an obvious question is whether similar receptors monitor salt and water balance in other vertebrates. A field in which avian studies have been of great fundamental value and ahead of mammalian studies is the photoperiodic control of reproduction. Therefore the control of reproductive cycles and reproductive behaviour occupied a large part of the symposium. B. K. Follett (University College of North Wales) described experiments done in collaboration with D. S. Farner (who led the final discussion) and colleagues at the University of Washington, Seattle, to test the Bünning hypothesis of biological time measurement; such a test would not have been possible had it not been for the development of a sensitive radioimmunoassay for avian luteinising hormone. Environmental factors were not overlooked and R. K. Murton (Monks Wood Experimental Station) explained the way in which ecological adaptation will have to be explained in terms of control mechanisms. But natural environmental factors were not the only type considered and A. S. Cooke, also from Monks Wood, produced clear evidence that some pesticides do have a scrious effect on an important reproductive process-formation of the egg shell.

Malcolm Peaker, who organised the symposium for the Zoological Society, explained that the programme had been arranged to cover avian physiology in its widest sense and to bring together those working on domesticated birds, as a part of poultry research, and those working on wild species. Thus this probably unique occasion for British ornithological science attracted a large audience with diverse basic interests, from field ornithology to neurophysiology and aeronautics to animal behaviour.

DRUG RESISTANCE

\section{Bacterial Ingenuity}

THE resistance to penicillin shown by many bacteria is in general mediated by a variety of penicillinases ( $\beta$ lactamases) which break the lactam ring of penicillin rendering it inactive. Semi-synthetic penicillins, such as methicillin and carbenicillin, have altered side chains making them far less sensitive to penicillinase action. The bacteria, as usual, have proved equal to the challenge.

In 1961, strains of Staphylococcus aureus resistant to methicillin appeared (Jevons, Br. med. J., 1, 124; 1961) only a year after introduction of the drug. On investigation, this resistance did not seem to be mediated by the appropriate penicillinase (methicillinase) (Seligman, Nature, 209, 994; 1966).

A component of the resistance to carbenicillin and other penicillins shown by another important wound pathogen, Pseudomonas aeruginosa carrying the $\mathbf{R}$ factor RP1 (Lowbury et al., Lancet, ii, 448; 1969), has now also been conclusively demonstrated not to involve a $\beta$ lactamase (Curtis et al., J. gen. Microbiol., 79,$163 ; 1973)$. The $\beta$ lactamase (IIIa) specified by this $\mathbf{R}$ factor hydrolyses carbenicillin relatively poorly, hardly sufficiently to account for the more than 1,000 times greater resistance shown by these strains. The Bristol group of Curtis et al. have now produced a mutant $\mathbf{R}$ factor which no longer codes for the $\beta$ lactamase but still confers appreciable resistance to carbenicillin and the other penicillins. Resistance to the other antibiotics specified by the $\mathbf{R}$ factor remained unchanged.

The mutant plasmid could not be reverted to $\beta$ lactamase production by further nitrosoguanidine treatment and no spontaneous mutants have been obtained, so it is probable that the $\beta$ lactamase genes have been deleted. This relatively high level of residual resistance is not due to a chromosomal gene as it disappears when the cells are cured of the plasmid by dodecyl sulphate treatment, neither is it due to a change in the levels of penicillinase Ia production by the plasmid-less cell.

The mutant $\mathbf{R}$ factor has been transferred to Escherichia coli $\mathrm{K} 12$ in which it also expresses a similar resistance pattern.

Penicillin resistance mediated by penicillinases was relatively straightforward to overcome by building new semi-synthetic penicillins, but even when the basis of this new type of resistance is uncovered it may prove difficult to circumvent, as it is much more likely to be due to structural and permeability changes in the cell itself. Methicillinresistant Staphylococcus aureus and carbenicillin-resistant Pseudomonas aeruginosa now represent a significant problem in the management of infected wounds.

\title{
Pole Tide in the North Sea-Baltic Area
}

As Lord Kelvin pointed out in 1876, a wobble of the Earth will induce an oceanic tide. In the case of the Chandler wobble, now known to have a period of about 14 months, the induced tide (pole tide) has a calculated equilibrium amplitude of only about $0.5 \mathrm{~cm}$, but is important because, if really equilibrium in form, it will lengthen the period of the wobble by about a month compared with the period for an Earth without oceans. Moreover, the existence of such a pole tide raises the possibility that the oceans may be a primary sink for the wobble energy; and in so far as excitation and decay are related problems, any information on modes of dissipation could throw light on the vexed question of the excitation mechanism of the Chandler wobble.

Measurement of the pole tide is difficult because of the high background noise in sea level, and so reported determinations are few. The most successful result was obtained many years ago by Haubrich and Munk (J. geophys. Res., 64, 2373; 1959), who found a fairly prominent peak in the sea level spectrum at about 0.84 cycles a year, corresponding to a period of $1.19 \mathrm{yr}$ (that is, about 14 months). Since then, however, the problem has attracted little attention except that data published by Maksimov and Karklin (Dokl. Acad. Nauk SSSR, $161,9 ; 1965)$ suggest a larger than equilibrium pole tide in the Baltic.

In Nature Physical Science next Monday (December 17), Miller and Wunsch report a further attempt to measure the pole tide, this time based on all available sea level records of more than $25 \mathrm{yr}$ duration published by the Association d'Oceanographie Physique. Most of the examined records were ambiguous; in most cases the pole tide did not rise above the background, and where it did it was weak. For records from the North Sea-Baltic area, however, the search was more successful, the pole tide being clearly visible at a frequency of 0.84 cycles a year. Moreover, Miller and Wunsch have also been able to show that there are systematic variations in the amplitude of the tide, from $0.7 \mathrm{~cm}$ at the southern end of the Netherlands coast to $1.8 \mathrm{~cm}$ at the northern end. In general, amplitudes rise markedly going eastwards from the North Sea into the Baltic and the Gulf of Bothnia, although the values in the constricted waters of the Kattegat are much lower than the general trend. 